\title{
Activation of corticotropin-releasing factor receptor 1 aggravates dextran sodium sulphate-induced colitis in mice by promoting M1 macrophage polarization
}

\author{
HONG CHEN $^{1}$, HAITAO SHI ${ }^{2}$, YAPING LIU ${ }^{1}$, XIAOYANG REN ${ }^{1}$, \\ SHUIXIANG $\mathrm{HE}^{1}$, XINMING $\mathrm{CHANG}^{1}$ and YAN YIN ${ }^{1}$ \\ ${ }^{1}$ Department of Gastroenterology, First Affiliated Hospital of Xi'an Jiaotong University, Xi'an, \\ Shaanxi 710061; ${ }^{2}$ Department of Gastroenterology, Second Affiliated Hospital of \\ Xi'an Jiaotong University, Xi'an, Shaanxi 710004, P.R. China
}

Received February 8, 2017; Accepted September 19, 2017

DOI: $10.3892 / \mathrm{mmr} .2017 .7909$

\begin{abstract}
The corticotropin-releasing factor (CRF) family is involved in modulating gastrointestinal motility, sensitivity and inflammation. CRF signalling exerts an important role in inflammatory bowel disease(IBD), predominantly by activating $\mathrm{CRF}$ receptors. The aim of the present study was to investigate the function of CRF receptor 1 (CRF-R1) in the development of mucosal inflammation induced by dextran sulphate sodium (DSS) and the underlying mechanism. Consecutive administration of CRF or CP154526 was used to activate or block the CRF-R1 in DSS-treated mice. Colonic inflammation was evaluated by determining the Disease Activity Index (DAI) and histology score. CRF-R1 expression was proportional to the DAI, the histology score and the number of macrophages. Activation of CRF-R1 aggravated mucosal inflammation by activating nuclear factor $(\mathrm{NF})-\kappa \mathrm{B}$ and subsequently increasing the expression levels of tumour necrosis factor (TNF)- $\alpha$ and interleukin (IL)-6. Inhibition of CRF-R1 decreased the expression level of CRF-R1, macrophage infiltration, NF- $\mathrm{B}$ activation, and TNF- $\alpha$ and IL-6 expression levels, ultimately alleviating the mucosal inflammation. Thus, CRF-R1 expression was proportional to the severity of DSS-induced colitis. Activation of CRF-R1 increased the DAI and histological scores of the colons from DSS-treated mice by promoting M1 macrophage polarization, demonstrated as increased $\mathrm{NF}-\kappa \mathrm{B}$ activation, and TNF- $\alpha$ and IL- 6 release. These results
\end{abstract}

Correspondence to: Dr Yan Yin or Dr Xinming Chang, Department of Gastroenterology, First Affiliated Hospital of Xi'an Jiaotong University, 277 Yanta West Road, Xi'an, Shaanxi 710061, P.R. China

E-mail: doctoryinyan@126.com

E-mail: cxm218@163.com

Key words: corticotropin-releasing factor receptor 1, ulcerative colitis, macrophage, nuclear factor- $\kappa \mathrm{B}$, tumour necrosis factor- $\alpha$, interleukin 6 provide evidence of the pro-inflammatory role of CRF-R1 in a DSS-induced ulcerative colitis (UC) model and a possible underlying mechanism, which may facilitate the elucidation of potential treatment approaches for UC.

\section{Introduction}

The corticotropin-releasing factor (CRF) family is well known for its role in the stress response. Psychosocial or environmental stressors induce or aggravate the development of inflammatory bowel disease (IBD) (1). During stress, CRF is released from the neurons of the paraventricular nucleus and activates the hypothalamic-pituitary-adrenal axis. Subsequently, glucocorticoids are produced in response to stress. In addition to central activation, CRF and associated peptides (urocortins) are released from regional sensory and sympathetic nerves, immune cells, and gut enteroendocrine cells to act locally. Along with its actions in the brain, CRF receptor signalling is involved in the pathogenesis of IBD by modulating gastrointestinal motility, sensitivity and inflammation (2-4).

In recent years, numerous studies have focused on the peripheral action of the CRF family on gastrointestinal inflammation (5). CRF and its receptors are located on immune cells in the blood, such as T cells, B cells and macrophages, as well as on colonic mucosal macrophages and enterochromaffin cells $(6,7)$. In a previous study, urocortin 1 and CRF receptor 1 (CRF-R1) expression levels were increased in colon biopsies from patients with ulcerative colitis (UC) compared with those of healthy subjects, indicating a possible peripheral role for CRF-R1 signalling in UC (8). CRF induced macrophages to release tumour necrosis factor (TNF)- $\alpha$, interleukin (IL)- $1 \beta$ and IL-6, and CRF-R1 antagonists blocked these effects (9). Furthermore, CFR-R1 knockout mice exhibited less inflammation than wild type mice in the dextran sulphate sodium (DSS)-induced UC mouse model (10). Based on these results, CRF-R1 may exert a pro-inflammatory role in intestinal inflammation. However, certain contrasting evidence supports an anti-inflammatory role for CRF-R1. CFR-R1 knockout did not reduce colon ulceration or edema (11), and a CRF deficiency increased the animals' susceptibility to developing 
colitis (12). CRF-R1 activation suppressed TNF- $\alpha$ release from lipopolysaccharide (LPS)-treated macrophages and inducible nitric oxide synthase from endothelial cells (13). Therefore, the pro- and anti-inflammatory roles of CRF-R1 remain controversial and may even be paradoxical. Studies are required to determine the role of CRF-R1 in colonic inflammation.

Macrophages are an important component of the pro-inflammatory response in murine models of colitis (14) and human IBD (15). Infiltration of activated macrophages into the lamina propria contributes to the development and progression of intestinal inflammation. CRF receptors are co-localized with macrophages and involved in their apoptosis (16). Furthermore, activation of CRF receptors augments cytokine production, such as TNF- $\alpha$ and IL-6, in LPS-induced macrophages (17), indicating that macrophages are a possible target of the CRF family to modulate inflammation.

Generally, nuclear factor $(\mathrm{NF})-\kappa \mathrm{B}$ controls various pro-inflammatory genes, chemokines and signalling enzymes, including TNF- $\alpha$, IL-6, IL-8 and IL-1 $\beta$, which are involved in regulating immune and inflammatory responses. $\mathrm{NF}-\kappa \mathrm{B}$ activation is induced in macrophages isolated from inflamed tissue specimens from patients with IBD (18), and the expression was correlated with the severity of colonic inflammation (19). CRF regulates the expression of the Toll-like receptor (TLR)-4 gene in macrophages and activates downstream effectors, such as $\mathrm{NF}-\kappa \mathrm{B}$ and pro-inflammatory cytokines, to induce an inflammatory reaction $(12,20)$. Therefore, regulation of $N F-\kappa B$ signalling may present as a potential therapeutic approach for IBD.

In the present study, the correlation between CRF-R1 expression and the disease severity was evaluated in a DSS-induced UC model. Subsequently, a CRF-R1 agonist and antagonist were used to investigate the effects of CRF-R1 on colitis. Furthermore, the modulatory effects of CRF-R1 on macrophages and the $\mathrm{NF}-\kappa \mathrm{B}$ signalling pathway were evaluated.

\section{Materials and methods}

Animals. A total of 56 specific pathogen-free, male BALB/c mice (weight, 18-22 g; Xi'an Jiaotong University Animal Centre, Xi'an, China) were randomly allocated into the following eight groups: DSS 0 group $(n=6)$, mice were administered normal drinking water; DSS 1 day group $(n=6)$, mice were administered 3\% DSS for 1 day; DSS 4 day group $(n=6)$, mice were administered $3 \%$ DSS for 4 consecutive days; DSS 7 day group $(n=6)$, mice were administered 3\% DSS for 7 consecutive days; normal group $(n=8)$, mice received saline $(0.2 \mathrm{ml})$ intraperitoneal (ip) injection daily and normal water drinking for 7 consecutive days; saline + DSS group $(n=8)$, mice received saline ip injection daily and 3\% DSS for 7 consecutive days; CRF + DSS group $(n=8)$, mice received $\mathrm{CRF}(30 \mu \mathrm{g} / \mathrm{kg})$ ip injection daily and 3\% DSS for 7 consecutive days; CP154526 + DSS group $(n=8)$, mice received CP154526 (10 mg/kg) ip injection daily and 3\% DSS for 7 consecutive days. All mice were housed under standard conditions, 4 mice per cage (temperature, $20-22^{\circ} \mathrm{C} ; 50-60 \%$ humidity, $12-\mathrm{h}$ light/dark cycle), with free access to standard rat chow and tap water. All experiments were performed in accordance with the United Kingdom Animals Act 1986 and were approved by the Ethical Committee of the Medical Faculty of Medicine of Xi'an Jiaotong University.

Animal model of UC. Mice were administered 3\% DSS (9011-18-1; molecular weight, 40,000; MP Biomedicals Inc., Santa Ana, CA, USA) as their sole source of drinking water for 7 consecutive days to develop UC model. Control mice received normal drinking water. Mice of normal group, saline + DSS group, CRF + DSS group and CP154526 + DSS group were sacrificed 7 days following the administration of DSS. Colon samples $(1.5 \mathrm{~cm})$ were collected from the anus. One third of the colon sample were rinsed in $4 \%$ paraformaldehyde for haematoxylin and eosin (H\&E) and immunohistochemical staining, and two thirds of the colon sample were rinsed in liquid nitrogen for reverse transcription-quantitative polymerase chain reaction (RT-qPCR), ELISA and western blot analysis. CRF (1151; R\&D Systems Inc., Minneapolis, MN, USA) was ip-injected into the DSS-treated mice at a dose of $30 \mu \mathrm{g} / \mathrm{kg}$ from the first day to the seventh day. CP154526 (PZ0100; Sigma-Aldrich; Merck KGaA, Darmstadt, Germany) was ip-injected into the DSS-treated mice at a dose of $10 \mathrm{mg} / \mathrm{kg}$ from the first day to the seventh day. Saline was ip-injected into the DSS-treated mice daily to serve as the control group. The CRF and CP154526 doses were determined according to our pilot experiment and previous studies (21-23).

In order to evaluate alterations in expression levels of CRF-R1 with the number of days of DSS administration, mice of DSS 0, DSS 1, DSS 4 and DSS 7 day groups were sacrificed on day $0,1,4$ and 7 days, respectively. Colon samples were collected for H\&E staining and RT-qPCR analysis as previously aforementioned.

Disease activity index (DAI) assessment. The DAI has been used to evaluate the severity of colitis, which is well correlated with the pathological findings in DSS-treated mice (24). The DAI is the combined score of weight loss, stool consistency and bleeding. Scores were defined for weight loss as follows: 0 , no loss; $1,1-5 \% ; 2,6-10 \% ; 3,11-15 \%$; and $4,>15 \%$. Scores for stools were as follows: 0 , normal; 2 , loose stool; 4 , diarrhoea. Bleeding scores were defined as follows: 0, no blood; 2 , presence; 4 , gross hematochezia. The DAI was scored from days 0 to 7 of DSS treatment.

Histopathological analysis. Colon sections were fixed overnight with $4 \%$ paraformaldehyde at $4^{\circ} \mathrm{C}$, and were then embedded in paraffin and serially sectioned. Samples were sliced into $4-\mu \mathrm{m}$ sections. Tissue sections were stained with hematoxylin (MHS16; Sigma-Aldrich; Merck KGaA; at room temperature for $20 \mathrm{~min}$ ) and eosin (230251; Sigma-Aldrich; Merck KGaA; at room temperature for $30 \mathrm{sec}$ ), and examined by a pathologist who was blinded to the experimental groups. The colonic histopathological score was assigned based on the extent and range of oedema, ulceration and infiltration of inflammatory cells, as described in a previous study (25). For immunohistochemistry, 4\% paraformaldehyde-fixed, paraffin-embedded tissues were sliced into $4-\mu \mathrm{m}$ slices. Non-specific antigens were blocked with $10 \%$ goat serum (SP-9001; Beijing Zhongshan Golden Bridge Biotechnology Co., Ltd., Beijing, China) at room temperature for $15 \mathrm{~min}$ following antigen retrieval. Antigen retrieval was performed 
in $0.01 \mathrm{M}$ citrate buffer ( $\mathrm{pH}$ 6.0) for $10 \mathrm{~min}$ in boiling water in a microwave oven. Sections were then incubated overnight at $4^{\circ} \mathrm{C}$ with a cluster of differentiation (CD)68 primary antibody (BA3638; 1:200; Wuhan Boster Biological Technology, Ltd., Wuhan, China). A SPlink detection kit (SP-9001; Beijing Zhongshan Golden Bridge Biotechnology Co., Ltd.) was used as a ready-to-use secondary antibody. The sections were incubated at $37^{\circ} \mathrm{C}$ for $30 \mathrm{~min}$. Positive binding was detected using diaminobenzidine (ZLI9033; Beijing Zhongshan Golden Bridge Biotechnology Co., Ltd.) staining for 1-3 min. Counterstaining with hematoxylin was performed at room temperature for $1 \mathrm{~min}$. The sections were examined using an optical microscopy (Olympus Corporation, Tokyo, Japan).

Measurement of CRF-R1 expression levels with RT-qPCR. Total RNA was extracted from the colon tissue using TRIzol ${ }^{\mathrm{TM}}$ reagent (15596026; Invitrogen; Thermo Fisher Scientific, Inc., Waltham, MA, USA) according to the manufacturer's instructions. RT was performed using a First Strand cDNA Synthesis kit (K1612; Thermo Fisher Scientific, Inc.). cDNA (1 $\mu 1)$ served as the template and was amplified by qPCR using the $2 \mathrm{X}$ SYBR-Green qPCR mix (PC3302; Aidlab Biotechnologies, Co., Ltd., Beijing, China) and $1 \mu \mathrm{l}$ sense and antisense primers in a final reaction volume of $25 \mu 1$ to quantify the cDNA contents. The primer sequences were as follows: CRF-R1 sense, TCTATGAGGAAGGAGTGG and antisense, GGCTTA GTTAGTTAGTTGTC; and $\beta$-actin sense, ACCACACCTTCT ACAATGAG and antisense, ACGACCAGAGGCATACAG. The size of the amplified products was expected to be $196 \mathrm{bp}$. The reactions were performed in triplicate to allow for statistical evaluation, and RT-qPCR was performed using an ABI-Prizm 7000 Real-Time PCR, DNA Thermal Cycler using the following cycling parameters: Pre-amplification cycle (denaturation at $95^{\circ} \mathrm{C}$ for $5 \mathrm{~min}$ ), 40 cycles of amplification (denaturation at $95^{\circ} \mathrm{C}$ for $15 \mathrm{sec}$, annealing at $60^{\circ} \mathrm{C}$ for $30 \mathrm{sec}$ and extension at $72^{\circ} \mathrm{C}$ for $30 \mathrm{sec}$ ) and a final extension step at $55^{\circ} \mathrm{C}$ for $30 \mathrm{sec}$. No by-products were present in the reaction, as indicated by the dissociation curve provided at the end of the reaction. The $2^{-\Delta \Delta \mathrm{Cq}}$ method was used to calculate the relative gene expression levels.

Measurement of CRF-R1 and p65 NF- $\kappa$ B phosphorylation using western blot analysis. Total protein was extracted from the colon tissue samples via radio immunoprecipitation assay (RIPA) lysis buffer (WB-0071; Beijing Dingguo Changsheng Biotechnology Co., Ltd., Beijing, China). Protein samples were mixed with sample buffer $(5 \mathrm{X})$ and denatured by boiling. For immunoblots, proteins were separated by sodium dodecyl sulphate polyacrylamide gel electrophoresis on a $10 \%$ polyacrylamide gel. Samples were electrophoresed at 80-100 V for $2 \mathrm{~h}$ until the dye migrated to the bottom of the gel. The proteins were transferred to polyvinylidene difluoride membranes and the membranes were blocked in blocking buffer (5\% non-fat dry milk) for $2 \mathrm{~h}$ at room temperature, and incubated with primary antibodies against CRF-R1 (ab59023; 1:500; Abcam, Cambridge, MA, USA) or p-p65 $\mathrm{NF}_{-} \mathrm{B}$ (3031; 1:1,000; Cell Signaling Technology, Inc., Danvers, MA, USA) and $\beta$-actin (wl01845; 1:1,000; Wanlei Biotechnology, Co., Ltd., Shenyang, China) overnight at $4^{\circ} \mathrm{C}$. Membranes were then incubated with a horseradish peroxidase (HRP)-conjugated anti-goat
(SA00001-4; 1:7,000; Proteintech Group, Inc., Rosemont, IL, USA) and anti-rabbit (Wla023a; 1:8,000; Wanlei Biotechnology, Co., Ltd.) secondary antibody for $1 \mathrm{~h}$ at room temperature. The proteins were visualized with a chemiluminescent substrate (WBULS0100; EMD Millipore, Billerica, MA, USA) using an imaging system (Bio-Rad Laboratories Inc., Hercules, CA, USA).

Measurement of the TNF- $\alpha$ and IL-6 expression levels using ELISA. A murine cytokine ELISA kit (K00164 and K00041; Shanghai Yeyuan Biology Science \& Technology, Shanghai, China) was used to measure the production of cytokines, TNF- $\alpha$ and IL-6 in the colon tissue samples. The samples were homogenized with saline (1:9) in a digital homogenizer, and the supernatant was obtained by centrifugation at $1,500 \mathrm{x} \mathrm{g}$, $4^{\circ} \mathrm{C}$ for $10 \mathrm{~min}$. All reagents were brought to room temperature. The plate was set for standard wells (6 wells) and testing sample wells (31 wells). Subsequently, each standard well was added $50 \mu \mathrm{l}$ standard sample; each testing sample well was added $10 \mu \mathrm{l}$ testing sample and then $40 \mu \mathrm{l}$ sample diluent. The plate was gently agitated for $1 \mathrm{~min}$ and was then incubated at room temperature for $2 \mathrm{~h}$. Subsequently, $100 \mu \mathrm{l}$ HRP-labeled antibody was added to each well and was incubated at $37^{\circ} \mathrm{C}$ for $1 \mathrm{~h}$. After washing, chromogen solution A (50 $\mu \mathrm{l})$ and chromogen solution B $(50 \mu \mathrm{l})$ were added to each well and the reaction was terminated with $50 \mu$ stop solution. The TNF- $\alpha$ and IL-6 concentrations were calculated based on the optical density at a wavelength of $450 \mathrm{~nm}$ using a microtiter plate reader (Bio-Rad Laboratories, Inc.).

Statistical analysis. Data processing and statistical analysis were performed using SPSS 18.0 statistical software (SPSS, Inc., Chicago, IL, USA) and all data are expressed as the means \pm standard error of the means. The data from multiple groups were compared using one-way analysis of variance, and comparisons between two groups were performed with the least significant difference (LSD) test. $\mathrm{P}<0.05$ was considered to indicate a statistically significant difference.

\section{Results}

CRF-RI expression level was increased in the colon samples from DSS-treated mice. To observe the expression level of CRF-R1 at different days of DSS administration, mice of DSS 0 , DSS 1, DSS 4 and DSS 7 day groups were sacrificed on day $0,1,4$ and 7 respectively, and samples from the colons were obtained to detect CRF-R1 expression levels and mucosal inflammation. As the number of days that the animals received DSS-containing water increased, the mucosal inflammation was significantly aggravated, which manifested as increased mucosa oedema, ulceration and infiltration of inflammatory cells. The expression level of the CRF-R1 mRNA increased as the number of days the animals were treated with DSS increased (Fig. 1A), and was correlated with the degree of mucosal inflammation.

To evaluate the effect of CRF-R1 agonist and antagonist on expression level of CRF-R1 of DSS induced UC mice, mice of normal group, saline + DSS group, CRF + DSS group and CP154526 + DSS group were sacrificed 7 days after commencing DSS administration. CP154526, a 
Table I. Effect of CRF-R1 on DAI of DSS-induced mice.

\begin{tabular}{lccccc}
\hline Groups & Day 1 & Day 3 & Day 5 & Day 6 & Day 7 \\
\hline Normal group & $0.000 \pm 0.000$ & $0.042 \pm 0.118$ & $0.083 \pm 0.154$ & $0.042 \pm 0.118^{\mathrm{a}}$ & $0.083 \pm 0.153^{\mathrm{a}}$ \\
Saline + DSS group & $0.125 \pm 0.173$ & $0.750 \pm 0.636^{\mathrm{b}}$ & $1.833 \pm 1.141$ & $2.917 \pm 1.318$ & $3.417 \pm 0.886$ \\
CRF + DSS group & $0.167 \pm 0.252$ & $0.916 \pm 0.463^{\mathrm{b}}$ & $2.082 \pm 0.635^{\mathrm{a}}$ & $3.500 \pm 0.535^{\mathrm{a}}$ & $3.917 \pm 0.155^{\mathrm{a}}$ \\
CP154526 + DSS group & $0.124 \pm 0.171$ & $0.750 \pm 0.344^{\mathrm{b}}$ & $1.417 \pm 0.497^{\mathrm{a}}$ & $2.417 \pm 0.683^{\mathrm{a}}$ & $3.084 \pm 0.463^{\mathrm{a}}$ \\
\hline
\end{tabular}

As the number of days the animals received DSS-containing water increased, the DAI score increased. CRF increased the DAI score and CP154526 decreased the DAI score compared with the saline + DSS group. ${ }^{a} \mathrm{P}<0.05$ vs. the saline + DSS group; ${ }^{\text {b }}<0.05$ vs. normal group. CRF, corticotropin-releasing factor; CRF-R1, corticotropin-releasing factor receptor 1; DAI, disease activity index; DSS, dextran sulphate sodium.

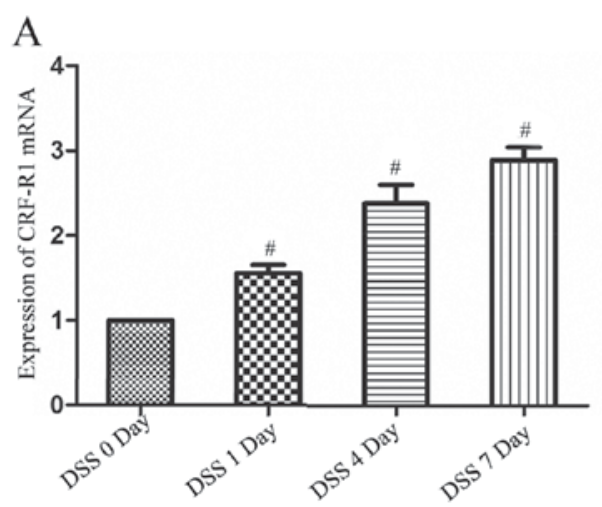

C

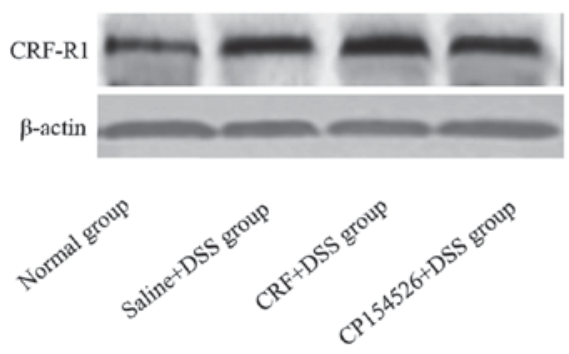

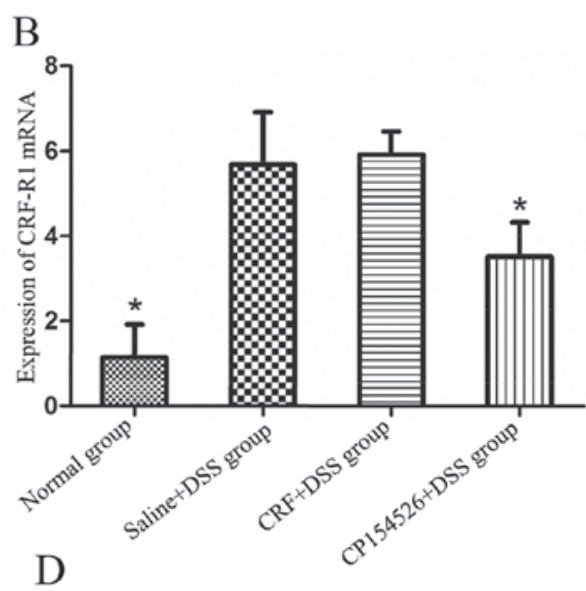

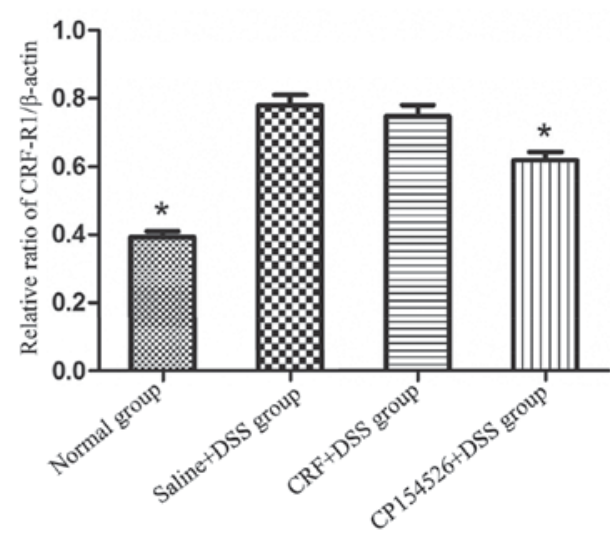

Figure 1. CRF-R1 expression level was increased in the colon samples of the DSS-treated mice. (A) Expression level of CRF-R1 mRNA in the colon samples from the DSS-treated mice increased as the number of days of DSS treatment increased. The ip injections of $10 \mathrm{mg} / \mathrm{kg} \mathrm{CP} 154526$ ( $\mathrm{n}=8)$ significantly decreased the CRF-R1 (B) mRNA and (C and D) protein expression levels in the colon samples from the DSS-treated mice. However, the ip injections of $30 \mu \mathrm{g} / \mathrm{kg}$ CRF $(\mathrm{n}=7)$ did not alter the expression level of CRF-R1. ${ }^{*} \mathrm{P}<0.05$ vs. the DSS-treated mice on day $0 .{ }^{*} \mathrm{P}<0.05$ vs. saline + DSS group. CRF, corticotropin-releasing factor; CRF-R1, corticotropin-releasing factor receptor 1; DSS, dextran sulphate sodium.

CRF-R1 antagonist, was ip-injected at a dose of $10 \mathrm{mg} / \mathrm{kg}$ for 7 consecutive days and significantly decreased the expression levels of the CRF-R1 mRNA and protein in the DSS-treated mice compared with that in the controls. Furthermore, CRF, a CRF-R1 agonist, was ip-injected at a dose of $30 \mu \mathrm{g} / \mathrm{kg}$ for 7 consecutive days and marginally increased the expression level of the CRF-R1 mRNA and protein in the DSS-treated mice compared with that in the controls (Fig. 1B-D).

Effect of CRF-RI on DSS-induced colitis. The ip injections of $\mathrm{CRF}$ aggravated the colonic mucosal inflammation induced by DSS on the seventh day compared with the controls. By contrast, the ip injections of CP154526 alleviated the colonic mucosal inflammation. The DAI (Table I) and histopathological score (Fig. 2) were significantly increased in the CRF-treated group and decreased in the CP154526-treated group compared with those in the saline + DSS controls. One mouse from the CRF-treated group succumbed on the seventh day of DSS treatment.

Macrophages were diffusely located throughout the lamina propria and submucosal layer in the colon samples of the DSS-treated mice, which appeared as brown 
A
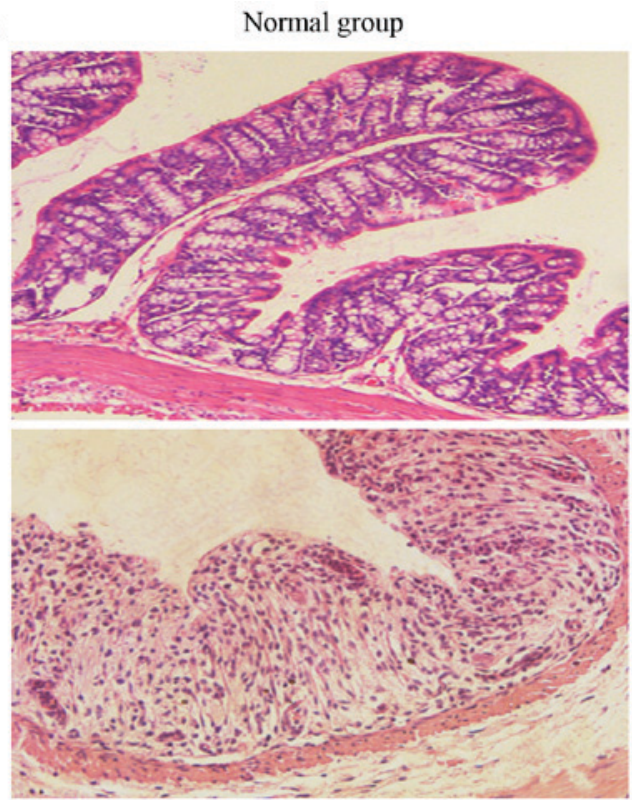

CRF+DSS group

B

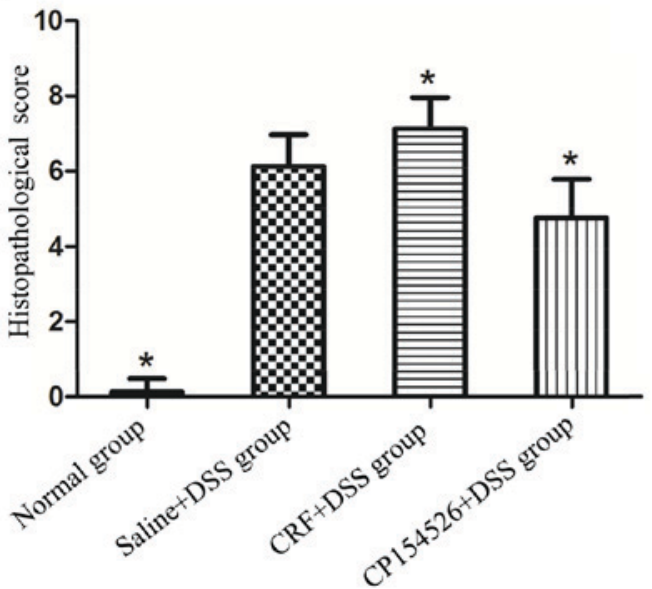

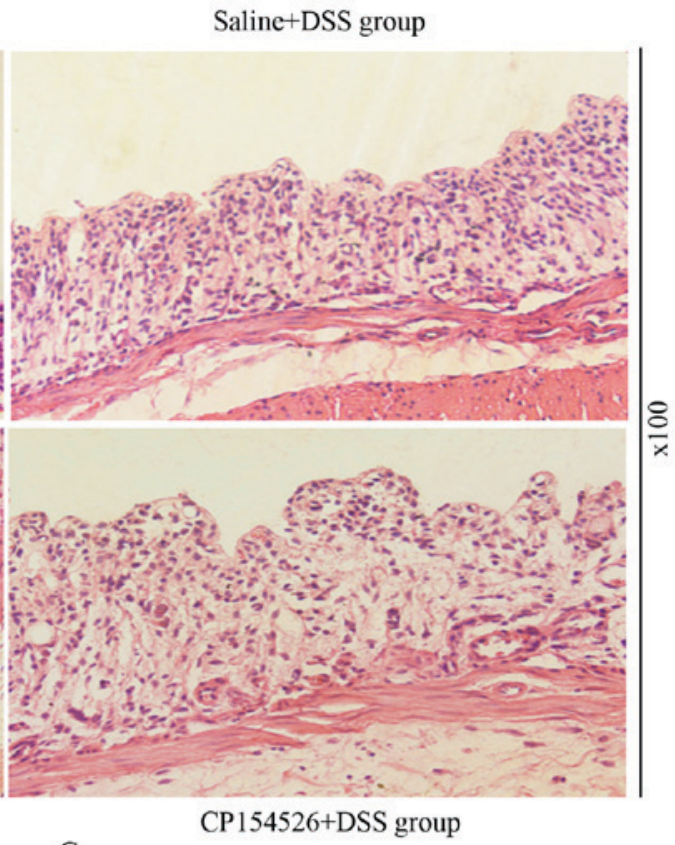

$\mathrm{C}$
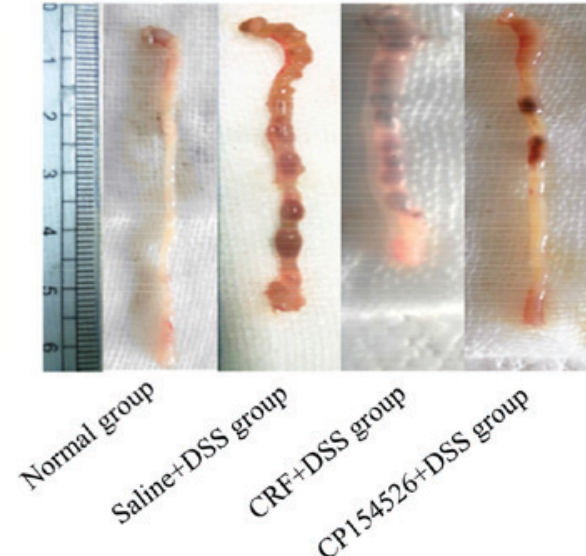

Figure 2. Effect of CRF-R1 on DSS-induced colitis. The ip injection of CRF aggravated DSS-induced colitis, (A) exhibited by increased mucosa oedema, ulcerations and infiltration of inflammatory cells (magnification, x100), (B) evaluated by histopathological score and (C) shortened colon. By contrast, the ip injection of CP154526 alleviated DSS-induced colitis. ${ }^{*}<0.05$ vs. saline + DSS group. CRF, corticotropin-releasing factor; CRF-R1, corticotropin-releasing factor receptor 1; DSS, dextran sulphate sodium; ip, intraperitoneal.

cytoplasmic staining. The DSS treatment significantly increased the number of CD68-positive macrophages compared with that in the controls, particularly on the seventh day. CP154526 decreased the infiltration of macrophages in the colons of the DSS-treated mice; however, CRF induced a marginal increase in the number of macrophages (Fig. 3).

Phosphorylation of p65 NF- $\kappa B$ was involved in the effect of CRF-RI on DSS-induced mucosal inflammation. The effect of CRF-R1 on NF- $\kappa$ B activation was examined by western blot analysis to investigate the mechanism by which CRF-R1 modulates inflammation in the DSS-treated mice. CRF, the CRF-R1 agonist, increased the level of p-p65 NF- $\kappa \mathrm{B}$ in the colon compared with that in the saline-injected controls. However, CP154526, the CRF-R1 antagonist, decreased the level of p-p65 NF- $\kappa \mathrm{B}$ in the colon (Fig. 4). Based on the results, NF- $\mathrm{KB}$ activation was involved in the effect of CRF-R1 on DSS-induced colitis.
Activation of CRF-RI increased the expression levels of inflammatory cytokines in the DSS-treated mice. The ip injections of CRF increased the expression levels of TNF- $\alpha$ and IL-6 in the colon compared with that in the saline + DSS controls. By contrast, the ip injections of CP154526 decreased the expression levels of TNF- $\alpha$ and IL-6. Furthermore, the TNF- $\alpha$ and IL-6 expression levels were identified to be significantly different between these two groups (Fig. 5).

\section{Discussion}

Emerging evidence links the activation of CRF receptors with intestinal inflammation. As demonstrated in the present study, CRF-R1 expression levels were increased in the colons of the DSS-treated mice compared with those in the control mice and was proportional to the severity of colonic mucosal inflammation. CRF-R1 is closely associated with inflammatory disease. In the study of clostridium difficile toxin-A-induced colonic inflammation by Wlk et al (26), the expression levels 
A
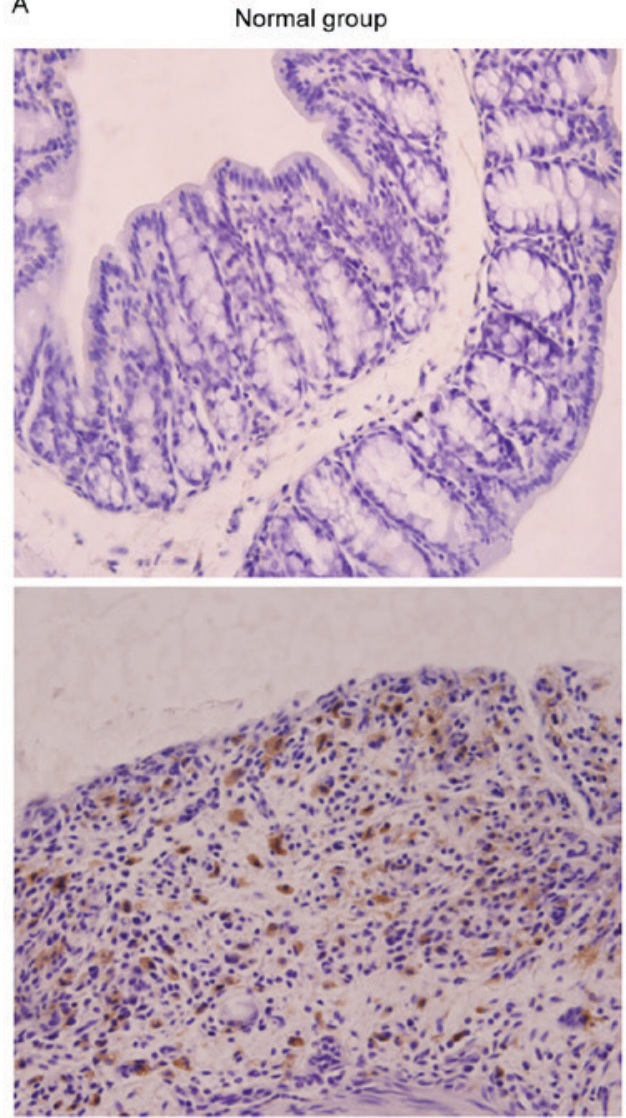

CRF+DSS group
Saline+DSS group
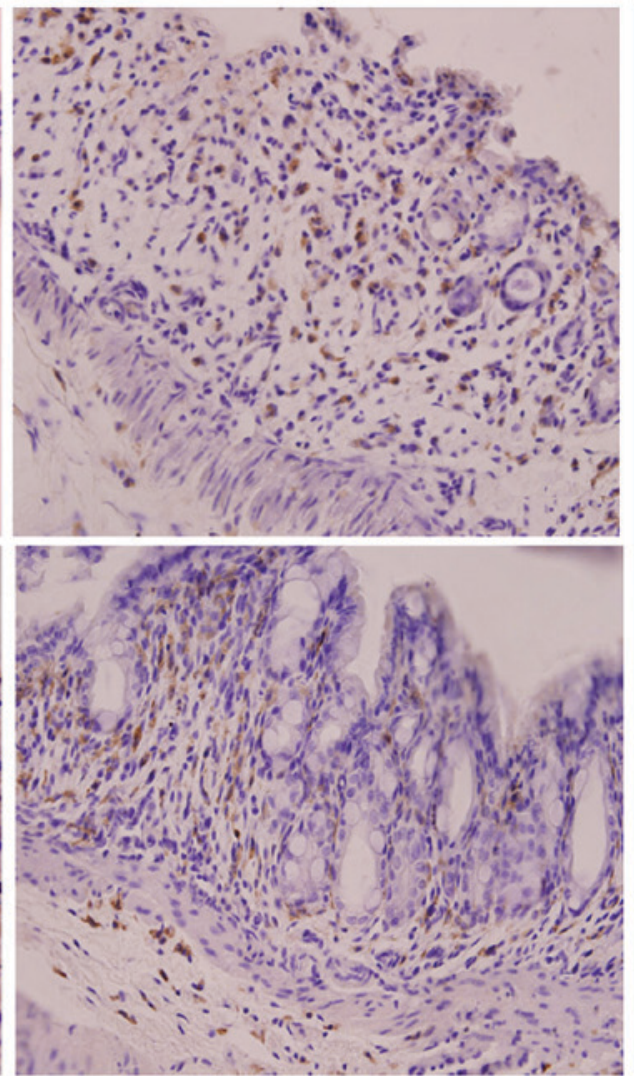

CP154526+DSS group

B

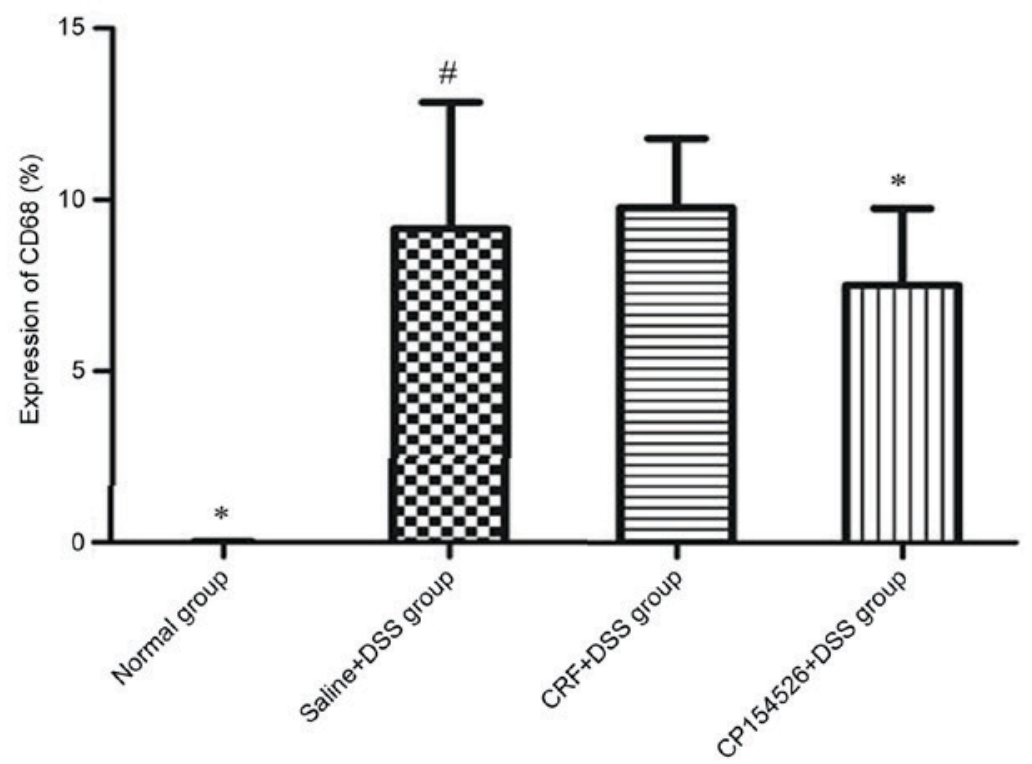

Figure 3. Effect of CRF-R1 on infiltration of macrophages in colon samples obtained from DSS-treated mice. (A) Compared with normal mice, a significant number of macrophages accumulated in the mucosa and submucosa of the colon on the seventh day of DSS treatment, which appeared as brown cytoplasmic staining. The ip injection of CP154526 significantly decreased the infiltration of macrophages in colon samples. (B) The ip injection of CRF slightly increased the number of macrophages in the colon samples. ${ }^{*} \mathrm{P}<0.05$ vs. saline + DSS group. ${ }^{"} \mathrm{P}<0.05$ vs. normal group. CRF, corticotropin-releasing factor; CRF-R1, corticotropin-releasing factor receptor 1; DSS, dextran sulphate sodium; ip, intraperitoneal; CD68, cluster of differentiation.

of CRF-R1 mRNA were increased in the mouse ileum. The synthesis and secretion of CRF-R1 from the colon is increased in patients with UC compared with in healthy subjects (27). In the current study, a DSS-induced UC model was used to further verify that the increased expression level of CRF-R1 was accompanied by aggravated ulcers, oedema and inflammatory cell infiltration in the colon. Furthermore, when colitis was alleviated, CRF-R1 expression levels decreased.

$\mathrm{CRF}$ is the predominant endogenous agonist that activates CRF-R1, which has a greater affinity for CRF-R1 than 
A

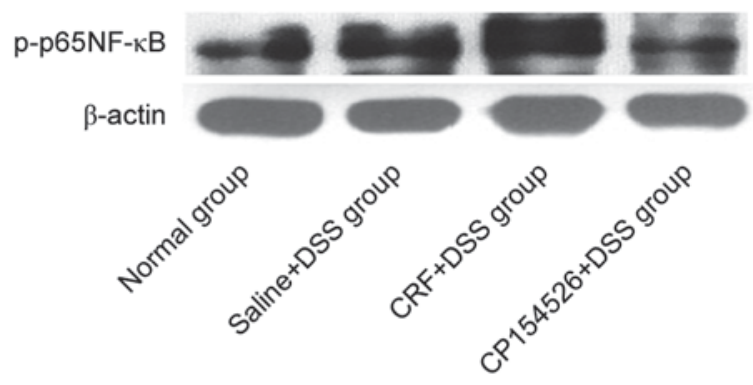

B

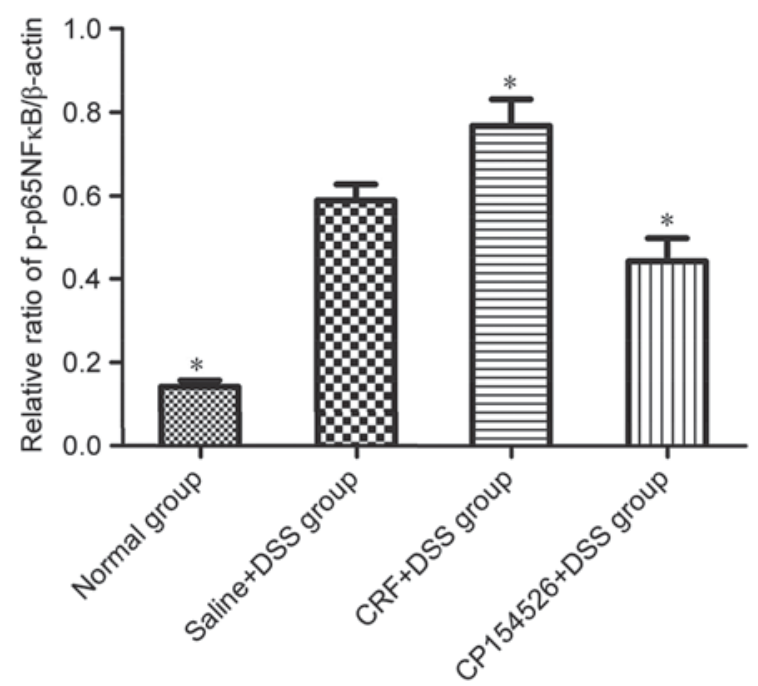

Figure 4. Effect of CRF-R1 on NF- $\kappa B$ phosphorylation in colon samples from DSS-treated mice. (A) The ip injection of CRF increased the expression level of p-p65 NF- $\mathrm{kB}$, and CP154526 decreased the expression level of p-p65 NF- $\mathrm{kB}$ in the colon samples compared with those from the saline + DSS group.

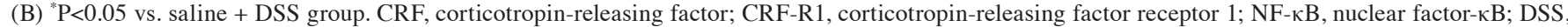
dextran sulphate sodium.

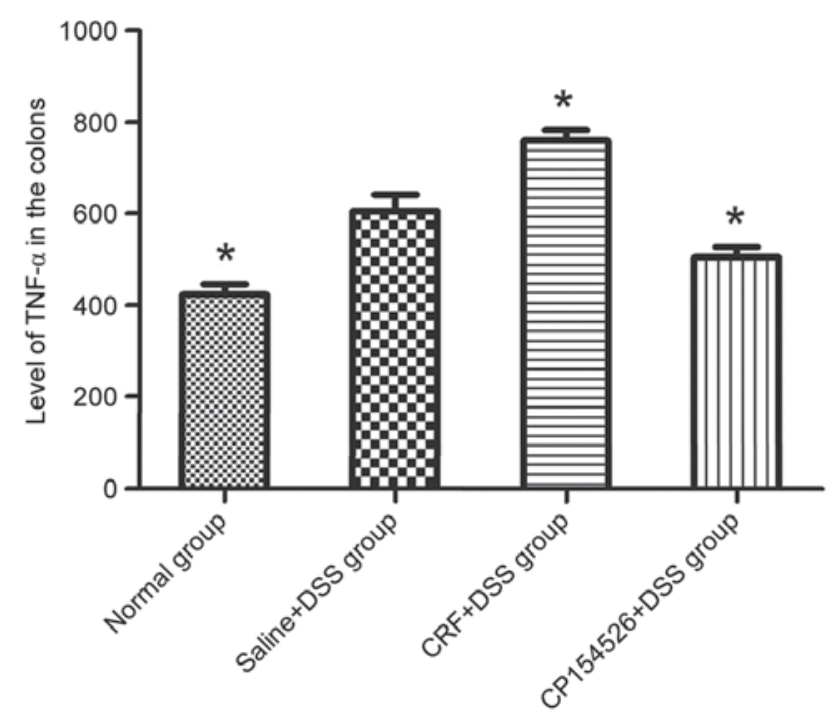

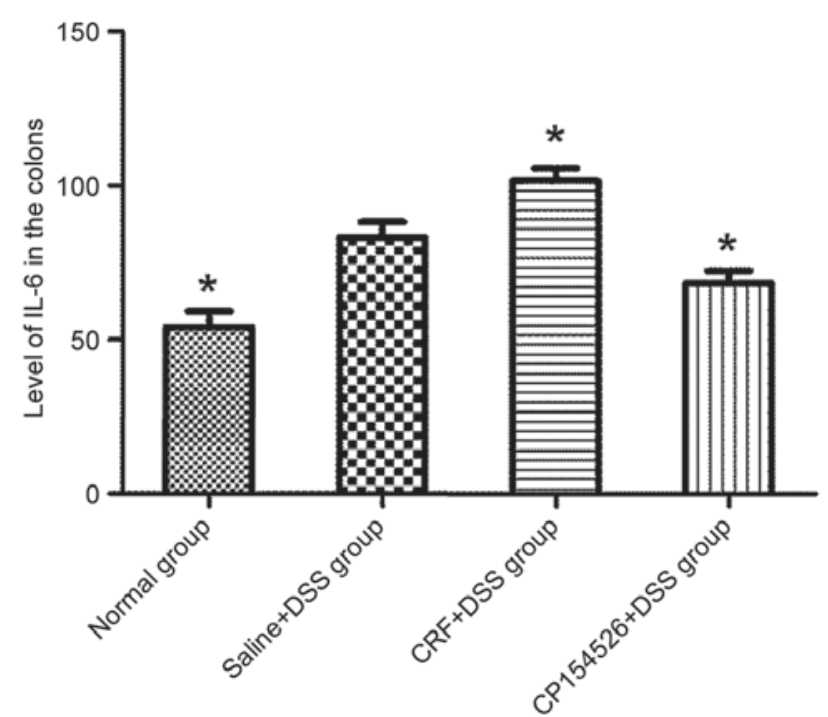

Figure 5. Effect of CRF-R1 on the (A) TNF- $\alpha$ and (B) IL-6 expression levels in the colon samples of the DSS-treated mice. The ip injection of CRF increased the expression levels of TNF- $\alpha$ and IL-6, and CP154526 decreased the expression levels of TNF- $\alpha$ and IL-6 in the colon compared with that in the saline + DSS group. " $\mathrm{P}<0.05$ vs. saline + DSS group. CRF, corticotropin-releasing factor; CRF-R1, corticotropin-releasing factor receptor 1; TNF- $\alpha$, tumor necrosis factor- $\alpha$; IL-6, interleukin 6; DSS, dextran sulphate sodium.

for CRF-R2. The CRF injections aggravated colitis in the DSS-treated mice compared with in the controls, as demonstrated by the increased DAI, colonic histopathology score and survival rate (one mouse succumbed). By contrast, injection of the specific CRF-R1 antagonist, CP154526 decreased the DAI, colonic histopathology score and expression level of CRF-R1 in the colon samples of the DSS-treated mice. These results are consistent with previous results obtained from $\mathrm{CRF} \mathrm{R}^{-/}$mice and the application of antalamin (another CRF-R1 antagonist) in DSS-treated mice $(10,28)$, confirming the pro-inflammatory role of CRF-R1 in the development of colitis. However, this finding contrasts with results from a recent study of rats expressing siCRF-R1, in which the histological scores and inflammatory cytokine levels were unchanged in the trinitrobenzene sulfonic acid-induced colitis model (11). The differences in these results may be attributed to the differences in the animal species, gene silencing methods and colitis models used.

Macrophages are important in modulating the inflammatory response in UC. According to the present study, macrophages were located in the mucosal and submucosal layers of the colon, and the level of CRF-R1 expression was proportional to macrophage infiltration in the colon samples of the DSS-treated mice. CRF-R1 is located in immune cells, 
such as lymphocytes, monocytes, and macrophages, in the lamina propria of the colon. The current results are similar to the data presented by Yuan et al (8) and Liu et al (10) who identified that the majority of CRF-R1 is co-localized with macrophages in an animal model of and patients with UC. Activation of CRF-R1 may induce the release of inflammatory factors from LPS-induced macrophages directly. In the present study, inhibition of CRF-R1 decreased the number of macrophages infiltrating the colon in the DSS-induced mice, which further illustrated that macrophages are a potential target for CRF-R1 to exert its pro-inflammatory effect $(17,20)$.

$\mathrm{NF}-\kappa \mathrm{B}$ is the crucial regulator of immune and inflammatory responses. Activation of the $\mathrm{NF}-\kappa \mathrm{B}$ signalling pathway in macrophages is crucial in the mechanism of UC, and the NF- $\kappa$ B expression level was increased in the colon of experimental animal models of colitis and patients with UC $(29,30)$. In the present study, p65-NF- $\kappa \mathrm{B}$ phosphorylation was increased in the colons of the DSS-treated mice compared with that in the controls. Activation of CRF-R1 by CRF increased p65-NF- $\mathrm{B}$ phosphorylation, and inhibition of CRF-R1 by CP154526 decreased its phosphorylation compared with that of the controls, which parallels the extent of colitis and expression levels of TNF- $\alpha$ and IL-6. Activation of NF- $\mathrm{BB}$ by CRF and associated peptides induces the release of inflammatory cytokines, such as the chemoattractants IL-8 and monocyte chemoattractant protein 1 in different cell types, including macrophages $(31,32)$. Thus, NF- $\kappa \mathrm{B}$ activation is involved in the effects of the CRF receptor on the immune reaction. Hence, the current study hypothesized that the pro-inflammatory effect of CRF-R1 on DSS-induced colitis correlated with the activation of p65 NF- $\kappa \mathrm{B}$.

Based on the results, CRF-R1 activation increased the expression levels of TNF- $\alpha$ and IL-6 in the colon samples of the DSS-treated mice. By contrast, inhibition of CRF-R1 by CP154526 decreased the expression levels of TNF- $\alpha$ and IL-6 in the colon tissues from the DSS-treated mice compared with that from the controls, accompanied by a decrease in NF- $\kappa \mathrm{B}$ phosphorylation and macrophage infiltration in the colon. As indicated by previous studies, activation of the CRF receptor increases the LPS-induced synthesis and release of TNF- $\alpha$ and IL- 6 from macrophages $(9,17)$. Furthermore, this role is associated with the regulation of TLR-2 and TLR-4, which activate macrophages to release pro-inflammatory cytokines by phosphorylating NF- $\mathrm{B}(20,12,33)$. Here, the current study verified that CRF-R1 exerted a pro-inflammatory role by activating $\mathrm{NF}-\kappa \mathrm{B}$, which in turn increased the expression levels of the pro-inflammatory cytokines, TNF- $\alpha$ and IL-6 in vivo. These findings may be associated with macrophage-mediated inhibition of CRF-R1, which may decrease the number of macrophages infiltrating the colon in the present study. Macrophage polarization balances immune reactions by releasing many inflammation-associated factors. M1 polarization is stimulated by interferon- $\gamma$ and TLRs, and these cells produce pro-inflammatory cytokines, such as IL-1 $\beta$, IL-6 and TNF- $\alpha$, to aggravate inflammation (34). By contrast, M2 polarization is stimulated by IL-4, and these cells produce anti-inflammatory cytokines to reverse or alleviate inflammation $(34,35)$. Together with previous studies on CRF-mediated activation of macrophages to induce inflammatory cytokine release in vitro, the current study hypothesizes that the pro-inflammatory role of CRF-R1 in DSS-induced colitis may be to regulate macrophage accumulation and activation. This action may contribute to M1 polarization, thus activating NF- $\kappa \mathrm{B}$ and releasing TNF- $\alpha$ and IL-6.

In conclusion, CRF-R1 expression levels were proportional to the severity of DSS-induced colitis. Activation of CRF-R1 aggravated inflammation, and inhibition of CRF-R1 ameliorates inflammation evaluated by the DAI and histological scores in the colon samples of the DSS-treated mice. These effects are reliant, at least in part, on the number of macrophages infiltrating the colon and trend to M1 polarization, exhibited as NF- $\kappa \mathrm{B}$ activation, and TNF- $\alpha$ and IL- 6 release. These results provide evidence for the pro-inflammatory role of CRF-R1 in a DSS-induced UC model and the possible underlying mechanism. Further studies are required to investigate markers of activation of M1 or M2 and the involved signal transduction pathway in CRF-R1-aggravated inflammation. Further investigation is required to understand whether antagonists that target CRF-R1 serve as a potential therapeutic approach for the treatment of UC.

\section{Acknowledgements}

The present study was supported by the Fundamental Research Funds for the Central Universities (grant no. 2015gjhz22) and the Shaanxi Science and Technology Research and Development Project (grant no. 2014K11-03-03-05).

\section{References}

1. Mawdsley JE and Rampton DS: Psychological stress in IBD: New insights into pathogenic and therapeutic implications. Gut 54: 1481-1491, 2005.

2. Taché Y, Kiank C and Stengel A: A role for corticotropin-releasing factor in functional gastrointestinal disorders. Curr Gastroenterol Rep 11: 270-277, 2009.

3. Taché Y and Bonaz B: Corticotropin-releasing factor receptors and stress-related alterations of gut motor function. J Clin Invest 117: 33-40, 2007.

4. Overman EL, Rivier JE and Moeser AJ: CRF induces intestinal epithelial barrier injury via the release of mast cell proteases and TNF- $\alpha$. PLoS One 7: e39935, 2012.

5. Buckinx R, Adriaensen D, Nassauw LV and Timmermans JP: Corticotrophin-releasing factor, related peptides, and receptors in the normal and inflamed gastrointestinal tract. Front Neurosci 5: $54,2011$.

6. Baker C, Richards LJ, Dayan CM and Jessop DS: Corticotropinreleasing hormone immunoreactivity in human $\mathrm{T}$ and $\mathrm{B}$ cells and macrophages: Colocalization with arginine vasopressin. J Neuroendocrinol 15: 1070-1074, 2003.

7. Kawahito Y, Sano H, Kawata M, Yuri K, Mukai S, Yamamura Y, Kato H, Chrousos GP, Wilder RL and Kondo M: Local secretion of corticotropin-releasing hormone by enterochromaffin cells in human colon. Gastroenterology 106: 859-865, 1994.

8. Yuan PQ, Wu SV, Elliott J, Anton PA, Chatzaki E, Million M and Taché Y: Expression of corticotropin releasing factor receptor type 1 (CRF1) in the human gastrointestinal tract and upregulation in the colonic mucosa in patients with ulcerative colitis. Peptides 38: 62-69, 2012.

9. Agelaki S, Tsatsanis C, Gravanis A and Margioris AN: Corticotropin-releasing hormone augments proinflammatory cytokine production from macrophages in vitro and in lipopolysaccharide-induced endotoxin shock in mice. Infect Immun 70: 6068-6074, 2002.

10. Liu Y, Fang X, Yuan J, Sun Z, Li C, Li R, Li L, Zhu C, Wan R, Guo $\mathrm{R}$, et al: The role of corticotropin-releasing hormone receptor 1 in the development of colitis-associated cancer in mouse model. Endocr Relat Cancer 21: 639-651, 2014.

11. Chang J, Adams MR, Clifton MS, Liao M, Brooks JH, Hasdemir B and Bhargava A: Urocortin 1 modulates immunosignaling in a rat model of colitis via corticotropin-releasing factor receptor 2. Am J Physiol Gastrointest Liver Physiol 300: G884-G894, 2011. 
12. Chaniotou Z, Giannogonas P, Theoharis S, Teli T, Gay J, Savidge T, Koutmani Y, Brugni J, Kokkotou E, Pothoulakis C and Karalis KP: Corticotropin-releasing factor regulates TLR4 expression in the colon and protects mice from colitis. Gastroenterology 139: 2083-2092, 2010.

13. Cantarella G, Lempereur L, Lombardo G, Chiarenza A Pafumi C, Zappla G and Bernadini R: Divergent effects of corticotropin releasing hormone on endothelial cell nitric oxide synthase are associated with different expression of CRH type 1 and 2 receptors. Br J Pharmacol 134: 837-844, 2001.

14. Dieleman LA, Palmen MJ, Akol H, Bloemena E, Peña AS, Meuwissen SG and Van Rees EP: Chronic experimental colitis induced by dextran sulphate sodium (DSS) is characterized by Th1 and Th2 cytokines. Clin Exp Immunol 114: 385-391, 1998.

15. Mahida YR, Wu K and Jewell DP: Enhanced production of interleukin 1-beta by mononuclear cells isolated from mucosa with active ulcerative colitis of Crohn's disease. Gut 30: 835-838, 1989.

16. Tsatsanis C, Androulidaki A, Dermitzaki E, Charalampopoulos I, Spiess J, Gravanis A and Margioris AN: Urocortin 1 and urocortin 2 induce macrophage apoptosis via CRFR 2. FEBS Lett 579: 4259-4264, 2005 .

17. Tsatsanis C, Androulidaki A, Dermitzaki E, Gravanis A and Margioris AN: Corticotropin releasing factor receptor 1 (CRF 1) and CRF 2 agonists exert an anti-inflammatory effect during the early phase of inflammation suppressing LPS-induced TNF-alpha release from macrophages via induction of COX-2 and PGE2. J Cell Physiol 210: 774-783, 2007.

18. Neurath MF, Pettersson S, Meyer zum Büschenfelde KH and Strober W: Local administration of antisense phosphorothioate oligonucleotides to the p65 subunit of NF-kappa B abrogates established experimental colitis in mice. Nat med 2: 998-1004, 1996.

19. Rogler G, Brand K, Vogl D, Page S, Hofmeister R, Andus T, Knuechel R, Baeuerle PA, Schölmerich J and Gross V: Nuclear factor kappaB is activated in macrophages and epithelial cells of inflamed intestinal mucosa. Gastroenterology 115: 357-369, 1998.

20. Tsatsanis C, Androulidaki A, Alissafi T, Charalampopoulos I, Dermitzaki E, Roger T, Gravanis A and Margioris AN: Corticotropin-releasing factor and the urocortins induce the expression of TLR4 in macrophages via activation of the transcription factors PU.1 and AP-1. J Immunol 176: 1869-1877, 2006.

21. Teitelbaum AA, Gareau MG, Jury J, Yang PC and Perdue MH: Chronic peripheral administration of corticotropin-releasing factor causes colonic barrier dysfunction similar to psychological stress. Am J Physiol Gastrointest Liver Physil 295: G452-G459, 2008.

22. Saito-Nakaya K, Hasegawa R, Nagura Y, Ito H and Fukudo S Corticotropin-releasing hormone receptor 1 antagonist blocks colonic hypersensitivity induced by a combination of inflammation and repetitive colorectal distension. Neurogastroenterol Motil 20: 1147-1156, 2008.

23. Castagliuolo I, Lamont JT, Qiu B, Fleming SM, Bhaskar KR Nikulasson ST, Kornetsky C and Pothoulakis C. Acute stress causes mucin release from rat colon: Role of corticotropin releasing factor and mast cells. Am J Physiol 271: G884-G892, 1996.
24. Cooper HS, Murthy SN, Shah RS and Sedergran DJ: Clinicopathologic study of dextran sulfate sodium experimental murine colitis. Lab Invest 69: 238-249, 1993.

25. Van der Sluis M, De Koning BA, De Bruijn AC, Velcich A, Meijerink JP, Van Goudoever JB, Büller HA, Dekker J, Van Seuningen I, Renes IB and Einerhand AW: Muc2-deficient mice spontaneously develop colitis, indicating that Muc2 is critical for colonic protection. Gastroenterology 131: 117-129, 2006.

26. Wlk M, Wang CC, Venihaki M, Liu J, Zhao D, Anton PM, Mykoniatis A, Pan A, Zacks J, Karalis K and Pothoulakis C: Corticotropin-releasing hormone antagonists possess anti-inflammatory effects in the mouse ileum. Gastroenterology 123: 505-515, 2002.

27. Saruta M, Takahashi K, Suzuki T, Torii A, Kawakami M and Sasano H: Urocortin 1 in colonic mucosa in patients with ulcerative colitis. J Clin Endocrinol Metab 89: 5352-5361, 2004.

28. Im E, Rhee SH, Park YS, Fiocchi C, Taché Y and Pothoulakis C: The corticotropin releasing hormone family of peptides regulates intestinal angiogenesis. Gastroenterology 138: 2457-2467, 2010.

29. Pandurangan AK, Ismail S, Saadatdoust $Z$ and Esa NM: Allicin alleviates dextran sodium sulfate-(DSS-) induced ulcerative colitis in BALB/c mice. Oxid Med Cell Longev 2015: 605208, 2015.

30. Pandurangan AK, Mohebali N, Mohd EN, Looi CY, Ismail S and Saadatdoust Z: Gallic acid suppresses inflammation in dextran sodium sulfate-induced colitis in mice: Possible mechanisms. Int Immunopharmacol 28: 1034-1043, 2015.

31. Kokkotou E, Torres D, Moss AC, O'Brien M, Grigoriadis DE, Karalis $\mathrm{K}$ and Pothoulakis C: Corticotropin-releasing hormone receptor 2-deficient mice have reduced intestinal inflammatory responses. J Immunol 177: 3355-3361, 2006.

32. Moss AC, Anton P, Savidge T, Newman P, Cheifetz AS, Gay J, Paraschos S, Winter MW, Moyer MP, Karalis K, et al: Urocortin II mediates pro-inflammatory effects in human colonocytes via corticotropin-releasing hormone receptor 2 alpha. Gut 56: 1210-1217, 2007.

33. Hermoso MA, Matsuguchi T, Smoak K and Cidlowski JA: Glucocorticoids and tumor necrosis factor alpha cooperatively regulate toll-like receptor 2 gene expression. Mol Cell Biol 24: 4743-4756, 2004.

34. Murray PJ, Allen JE, Biswas SK, Fisher EA, Gilroy DW, Goerdt S, Gordon S, Hamilton JA, Ivashkiv LB, Lawrence T, et al: Macrophage activation and polarization: Nomenclature and experimental guidelines. Immunity 41: 14-20, 2014.

35. Van Welden S, De Vos M, Wielockx B, Tavernier SJ, Dullaers M, Neyt S, Descamps B, Devisscher L, Devriese S, Van den Bossche L, et al: Haematopoietic prolyl hydroxylase-1 deficiency promotes M2 macrophage polarization and is both necessary and sufficient to protect against experimental colitis. J Pathol 241: 547-558, 2017.

This work is licensed under a Creative Commons Attribution-NonCommercial-NoDerivatives 4.0 International (CC BY-NC-ND 4.0) License. 